\title{
Thermocapillary Effect in a Thermal Rheological Jet
}

\section{Zemei Tang ${ }^{*}$ and Wenrui Hu}

National Microgravity Laboratory/CAS, Institute of Mechanics, Chinese Academy of Sciences, Beijing 100080, China

*Corresponding author (zmtang@imech.ac.cn)

Communicated by E. Michaelides, Denton, TX, USA

\begin{abstract}
The process of die swell in polymer jets is an important feature within polymer processing and can be explained through a study of its rheological effects. The existence of a thermocapillary effect, driven by the gradient of its surface tension, should be considered when examining a thermal jet that has a non-uniform temperature distribution on its free surface, as in various polymer processings. Both the rheological effect and thermocapillary effect on die swell can be studied numerically through a finite element method as used on a two-dimensional and unsteady model, in which a Coleman-Noll secondorder fluid model is employed. The results show that the expanding angle depends on both the rheological property of the fluid and the pressure at the vessel exit. Although both the thermocapillary and the rheological effects contribute to the cross-section expansion of the fluid jet, the latter is more important in determining the expansion.
\end{abstract}

\section{Introduction}

Cross-section expansions of thermal jets have been observed in polymer processing. This process is called die swell and is an important phenomenon. The study of hydrodynamics $[1,2]$ is a necessity when it comes to understanding the mechanism behind the process of die swell. A die swell effect is usually explained by the rheological property of the liquid [3-5]. It is believed that the variance in normal stresses enlarges the cross section of the liquid jet. 
On the other hand, experiments have shown that the solutal capillary flow, which is induced by a surfactant present in the liquid, might be responsible for increasing the thickness of the liquid layer [6]. On the basis of a simplified physical model, the LLD theory for fluid jet coating on a moving solid was able to give a qualitative explanation as to how the thermo- or solutocapillary effect increased the thickness of the jet [7]. The process of a liquid (or melt) film of a relatively high temperature being ejected from a vessel, and moving to coat a moving solid belt, is determined by thermal hydrodynamic equations. The temperature gradient on the free surface of a thermal jet is the result of a heat transfer that occurs during the movement from a relatively high temperature melt region to a low temperature environment. As a result, thermocapillary flow is induced, and may enlarge the cross section of the film in polymer processing. The thermocapillary effect on the process of die swell was investigated analytically in cases involving Newtonian fluids [8-10]. However, the relationship involving non-Newtonian fluids is very complicated, and only has an analytical solution when we restrict to weak non-Newtonian models [11]. The analysis is based on the approximations of lubrication theory and the perturbation method provided by an expansion of the small parameter $\varepsilon=\frac{h_{o}}{\ell}$. The zero-order and high-order equations have been solved analytically in order to determine the underlying heat and flow processes.

The phenomenon of cross-section expansion, due to both the thermocapillary effect and the rheological properties inherent in the specific cases of expanding angles, was studied numerically for a non-Newtonian liquid jet film that was cast on a moving solid boundary [12]. In the study, both the rheological and the thermocapillary effects were found capable of enlarging the cross section of the liquid jet.

The cross-section expansion of the liquid jet depends on both the rheological properties of the fluid and the pressure that exists at the vessel's point of exit. This paper explains a model in which a liquid film was ejected from a die and coated on a moving solid wall. The different exit pressures were discussed numerically, so that the effect of the exit pressure on the expansion of the liquid jet could be analyzed, as well as the effect of the rheological properties that correlated to each moment of variance in exit pressure. The Coleman-Noll second-order fluids model is adopted in the present paper. As a model, it is usually used for polymers with weak elasticity, such as dilute polyacrylamide or polyethleneoxide solutions. The cross-section expansion, the flow field, and temperature distribution of the liquid film as it was ejected from the die have been simulated by a two-dimensional and unsteady model for the sake of simplicity. Both the rheological and the thermocapillary effects are considered in our governing equations and boundary conditions. 
The physical and mathematical models used to explain the behavior of the non-Newtonian liquid film jet are described in the following section. It must be noted that, since the height of the jet is small, the present work does not take the effect of gravity into account. Moreover, in this calculation all thermophysical properties are treated as constants. The numerical method is discussed in the third section, and an explanation of the simulation results is provided in the last two sections.

\section{Theoretical model for non-Newtonian fluids}

The model discussed in the present paper is shown in Figure 1, as it illustrates the liquid being ejected from a vessel and coating a moving solid belt. If a Cartesian coordinate system $(x, y, z)$ is adopted, and it is assumed that $\partial / \partial y=0$, then the liquid flows from the vessel exit at $x=0$ to a point at $x=\ell$, a specific distance downstream. It is important to note that the initial height $h_{o}$ of the liquid layer at $x=0$ is much smaller than the distance $\ell$. Additionally, the liquid temperature $T_{0}$ at point $x=0$ is higher than both the environmental gas temperature $T_{g}$ and the temperature $T_{\ell}$ at $x=\ell$. A solid, moving belt smoothly touches the right boundary of the vessel, and moves with the same velocity $u_{s}$ as the liquid at $z=0$.

The liquid used for the calculation is an incompressible fluid that maintains a constant level of viscosity and thermal diffusivity. The conservation relationships are written as

$$
\frac{\partial u}{\partial x}+\frac{\partial w}{\partial z}=0
$$

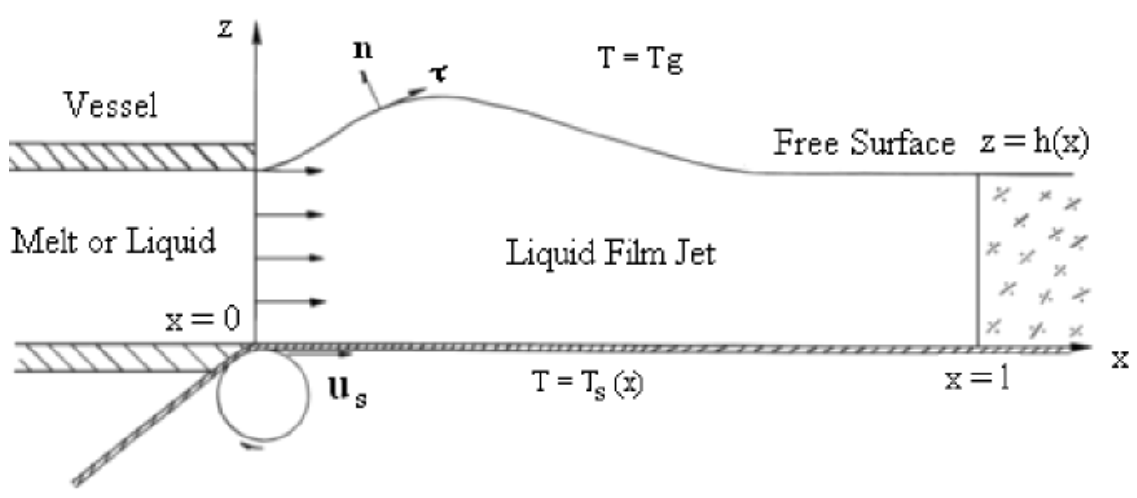

Figure 1 Schematic diagram of a liquid jet ejected from a vessel and coating on a moving solid boundary. 


$$
\begin{aligned}
& \rho \frac{\partial v}{\partial t}+\rho v \cdot \nabla v=-\nabla p+\mu \nabla^{2} v+\nabla \pi, \\
& \frac{\partial T}{\partial t}+v \cdot \nabla T=\kappa \nabla^{2} T .
\end{aligned}
$$

Here $\rho, p, \mu, \kappa$, and $T$ are the density, pressure, dynamic viscosity, heat diffusivity, and temperature of the liquid, respectively, $(u, 0, w)$ is the velocity vector, and $\pi_{x x}, \pi_{x z}, \pi_{z x}$, and $\pi_{z z}$ are the components of the stress tensor $\pi$.

The Coleman-Noll second-order fluids model, based on the Rivlin-Ericksen stress tensor, is used to describe non-Newtonian fluids $[10,11]$. In this case, we obtain

$$
\begin{aligned}
\pi_{x x}= & 2 \mu \frac{\partial u}{\partial x}+4 \alpha_{1}^{*}\left[\left(\frac{\partial u}{\partial x}\right)^{2}+\frac{1}{4}\left(\frac{\partial u}{\partial z}+\frac{\partial w}{\partial x}\right)^{2}\right] \\
& +2 \alpha_{2}^{*}\left[u \frac{\partial^{2} u}{\partial x^{2}}+w \frac{\partial^{2} u}{\partial x \partial z}+2\left(\frac{\partial u}{\partial x}\right)^{2}+\frac{\partial w}{\partial x}\left(\frac{\partial u}{\partial z}+\frac{\partial w}{\partial x}\right)\right] \\
\pi_{x z}= & \mu\left(\frac{\partial u}{\partial z}+\frac{\partial w}{\partial x}\right)+2 \alpha_{2}^{*}\left[u \frac{\partial}{\partial x}\left(\frac{\partial u}{\partial z}+\frac{\partial w}{\partial x}\right)+w \frac{\partial}{\partial z}\left(\frac{\partial u}{\partial z}+\frac{\partial w}{\partial x}\right)\right. \\
& \left.+2\left(\frac{\partial u}{\partial x} \frac{\partial u}{\partial z}+\frac{\partial w}{\partial x} \frac{\partial w}{\partial z}\right)\right] \\
\pi_{z z}= & 2 \mu \frac{\partial w}{\partial z}+4 \alpha_{1}^{*}\left[\left(\frac{\partial w}{\partial z}\right)^{2}+\frac{1}{4}\left(\frac{\partial u}{\partial z}+\frac{\partial w}{\partial x}\right)^{2}\right] \\
& +2 \alpha_{2}^{*}\left[w \frac{\partial^{2} w}{\partial z^{2}}+u \frac{\partial^{2} w}{\partial x \partial z}+2\left(\frac{\partial w}{\partial z}\right)^{2}+\frac{\partial u}{\partial z}\left(\frac{\partial u}{\partial z}+\frac{\partial w}{\partial x}\right)\right]
\end{aligned}
$$

where $\alpha_{1}^{*}$ and $\alpha_{2}^{*}$ are viscoelastic coefficients, subject to the conditions $\alpha_{1}^{*}>0$ and $\alpha_{2}^{*}<0$. The first normal stress difference is $-2 \alpha_{2}^{*}$. The second normal stress difference $\alpha_{1}^{*}+2 \alpha_{2}^{*}$ is generally small, and the condition

$$
\alpha_{1}^{*}+2 \alpha_{2}^{*}=0
$$

is assumed to be satisfactory for the purposes of the present paper.

The conditions for the physical boundaries of the liquid layer can be written as follows:

$$
\begin{aligned}
& z=0: \quad u=u_{s}, \quad w=0, \quad T=T_{s}(x)=T_{0}-\left(T_{0}-T_{\ell}\right)(x / \ell), \\
& z=h(x): \quad w=\frac{\partial h}{\partial t}+u \frac{\partial h}{\partial x},
\end{aligned}
$$




$$
\begin{aligned}
p= & \frac{2 \mu}{1+h^{\prime 2}}\left[h^{\prime 2} \frac{\partial u}{\partial x}-h^{\prime}\left(\frac{\partial w}{\partial x}+\frac{\partial u}{\partial z}\right)+\frac{\partial w}{\partial z}\right] \\
& +\frac{1}{1+h^{\prime 2}}\left[h^{\prime 2} \pi_{x x}-2 h^{\prime} \pi_{x z}+\pi_{z z}\right]-\sigma \frac{h^{\prime \prime}}{\left[1+h^{\prime 2}\right]^{3 / 2},} \\
\frac{-\left|\sigma_{T}^{\prime}\right|}{\sqrt{1+h^{\prime 2}}}\left(\frac{\partial T}{\partial x}+h^{\prime} \frac{\partial T}{\partial z}\right) & =\frac{\mu}{\left(1+h^{\prime 2}\right)}\left[2 h^{\prime}\left(\frac{\partial w}{\partial z}-\frac{\partial u}{\partial x}\right)+\left(1-h^{\prime 2}\right)\left(\frac{\partial u}{\partial z}+\frac{\partial w}{\partial x}\right)\right] \\
& +\frac{1}{\left(1+h^{\prime 2}\right)}\left[-h^{\prime} \pi_{x x}+\left(1-h^{\prime 2}\right) \pi_{x z}+h^{\prime} \pi_{z z}\right], \\
k \frac{\partial T}{\partial n}= & -H\left(T-T_{g}\right),
\end{aligned}
$$

where $h^{\prime}=\mathrm{d} h(x) / \mathrm{d} x, n$ is a unit used for normal vectors, $k$ is the heat conductivity of the liquid, and $H$ is the coefficient for heat transfer during the liquid-gas interface. The curvature of the free surface is

$$
\frac{1}{R_{c}}=\frac{h^{\prime \prime}(x)}{\sqrt[3]{1+h^{\prime 2}(x)}}
$$

and the radiation effect is negligible in Eq. (11). The boundary conditions (7) show a longitudinal temperature difference $T_{o}-T_{\ell}$, which may induce a temperature gradient on the free surface.

The flow is understood to be laminar, with the height of the liquid film registering a parabolic velocity distribution. The liquid film's boundary conditions at $x=0$ are as follows:

$$
x=0: \quad u=u_{0}\left[\frac{1}{4}-\left(\frac{z}{h_{0}}-\frac{1}{2}\right)^{2}\right], \quad w=0 ; \quad T=T_{0} .
$$

The boundary conditions at $x=\ell$ are

$$
x=\ell: \quad \frac{\partial u}{\partial x}=0, \quad \frac{\partial^{2} w}{\partial^{2} x}=0, \quad T=T_{\ell} .
$$

Boundary conditions (12) provide the kinetic energy at the vessel exit $x=0$ that drives the liquid jet into the region $x>0$.

\section{Numerical method}

The basic equations (1)-(3) are to be solved under the boundary conditions (7)-(13). The shape of the free boundary needs to be determined as part of 
the solution; it may be obtained by the method that adjusts iteratively the configuration of the free surface according to relevant velocity and pressure conditions at the free boundary.

The problem can also be solved by using an unsteady program, in which the initial free surface at $t=0$ is assumed to be of plane configuration, and the initial velocity $u$ at $x=0$ is derived from Eq. (12). The temperature difference $\Delta T=T_{o}-T_{l}$ is assumed to be zero at $t=0$; and in the remaining portion of the calculation, $T_{\ell}$ stays steady at $0^{\circ} \mathrm{C}$ but the temperature $T_{o}$ at the point $x=0$ is increased by a rate of $5^{\circ} \mathrm{C} / \mathrm{s}$ until it reaches the required temperature $T_{o}=T_{l}+\Delta T$, and then keeps constant. Both the field of flow and the temperature distribution that correspond to these initial conditions can be obtained numerically under the given boundary conditions (7$13)$.

The distribution of pressure on the free boundary of the jet can be calculated from the resulting velocity field, and the configuration of the free surface can be reconstructed by using an equation that establishes the equilibrium conditions for normal stress, i.e., Eq. (9). A steady solution can be reached when all the variables, in addition to the shape of the free surface, remain unchanged for a fixed temperature difference $\Delta T=T_{0}-T_{\ell}$.

Equations (1-3) can be written in non-dimensional forms, and the nondimensional quantities and parameters can be introduced as follows:

$$
\begin{aligned}
& \xi=\frac{x}{h_{o}}, \quad \eta=\frac{h}{h_{o}}, \quad \zeta=\frac{z}{h_{o}}, \quad \tau=\frac{t u_{*}}{h_{o}}, \quad U=\frac{u}{u_{*}}, \\
& W=\frac{w}{u_{*}}, \quad \Theta=\frac{T}{\Delta T}, \quad \alpha_{1}=\frac{\alpha_{1}^{*} u_{*}}{\mu h_{o}}, \quad \alpha_{2}=\frac{\alpha_{2}^{*} u_{*}}{\mu h_{o}}, \\
& \Pi=\frac{\pi h_{o}}{\mu u_{*}}, \quad R_{e}=\frac{\rho u_{*} h_{o}}{\mu}, \quad P_{e}=\frac{u_{*} h_{o}}{\kappa},
\end{aligned}
$$

where $\sigma$ is the surface tension and a typical velocity is represented by

$$
u^{*}=\left|\sigma_{\mathrm{T}}^{\prime}\right| \Delta T / \rho v .
$$

By defining the stream function $\psi$ and vorticity function $\omega$ as

$$
U=\frac{\partial \psi}{\partial z}, \quad W=-\frac{\partial \psi}{\partial x}, \quad \omega=\frac{\partial W}{\partial x}-\frac{\partial U}{\partial z},
$$

the non-dimensional equations can be expressed in terms of the stream function and the vorticity function as 


$$
\begin{aligned}
& \frac{\partial^{2} \psi}{\partial \xi^{2}}+\frac{\partial^{2} \psi}{\partial \zeta^{2}}=0 \\
& R_{e}\left(\frac{\partial \omega}{\partial \tau}+\frac{\partial \psi}{\partial \zeta} \frac{\partial \omega}{\partial \xi}-\frac{\partial \psi}{\partial \xi} \frac{\partial \omega}{\partial \zeta}\right)=\frac{\partial^{2} \omega}{\partial \xi^{2}}+\frac{\partial^{2} \omega}{\partial \zeta^{2}}+\frac{\partial^{2}\left(\Pi_{\xi \xi}-\Pi_{\zeta \zeta}\right)}{\partial \xi \partial \zeta} \\
& +\frac{\partial^{2} \Pi_{\xi \zeta}}{\partial \zeta^{2}}-\frac{\partial^{2} \Pi_{\xi \zeta}}{\partial \xi^{2}} \\
& P_{e}\left(\frac{\partial \Theta}{\partial \tau}+\frac{\partial \psi}{\partial \zeta} \frac{\partial \Theta}{\partial \xi}-\frac{\partial \psi}{\partial \xi} \frac{\partial \Theta}{\partial \zeta}\right)=\frac{\partial^{2} \Theta}{\partial \xi^{2}}+\frac{\partial^{2} \Theta}{\partial \zeta^{2}} .
\end{aligned}
$$

Problems that occur under the initial boundary conditions can be solved by a hybrid finite element method that includes fractional steps. The characteristic procedure is used for the convection operator, whereas the finite element method is used for the diffusion terms. The numbers of the cell of the calculated region are $121 \times 21$ in $\xi$ and $\zeta$ directions, respectively, which means that the domain dealt with in the computation was divided into 4800 triangular elements associated with 2541 nodes.

In the present simulation, the typical parameters are chosen as follows:

$$
\begin{aligned}
& h_{0}=1 \mathrm{~mm}, \quad \ell=50 \mathrm{~mm}, \quad u_{0}=64.2 \mathrm{~mm} / \mathrm{s}, \quad u_{s}=0.3 u_{0}, \\
& T_{0}=100^{\circ} \mathrm{C}, \quad \sigma=20.1 \mathrm{~g} / \mathrm{s}^{2}, \quad \sigma_{T}=-0.06 \mathrm{~g} / \mathrm{s}^{2} \mathrm{C},
\end{aligned}
$$

where $u_{0}=4 u^{*}$, and the related Reynolds number and Peclet number are, respectively, $R e=0.642, P e=2.03$.

The present program was used to calculate the thermocapillary convection in a square container. The results of these computations were compared with the results that have been obtained by Carpenter et al. [13] in their own research, and were found to be in correspondence [12]. This similarity of results validates the present computational program, showing that results given by the hybrid finite element method with fractional steps are compatible with those given by Carpenter.

\section{Simulation result}

The expanding angle of the thermal jet $\beta=\tan ^{-1}\left[\eta^{\prime}(0)\right]$ at the vessel exit $x=0$ is a sensitive parameter, and depends on both the non-Newtonian effect and the thermocapillary effect. Generally, the larger the rheological effect, the larger the expanding angle $\beta$. A non-dimensional parameter of the exit pressure is defined as follows: 


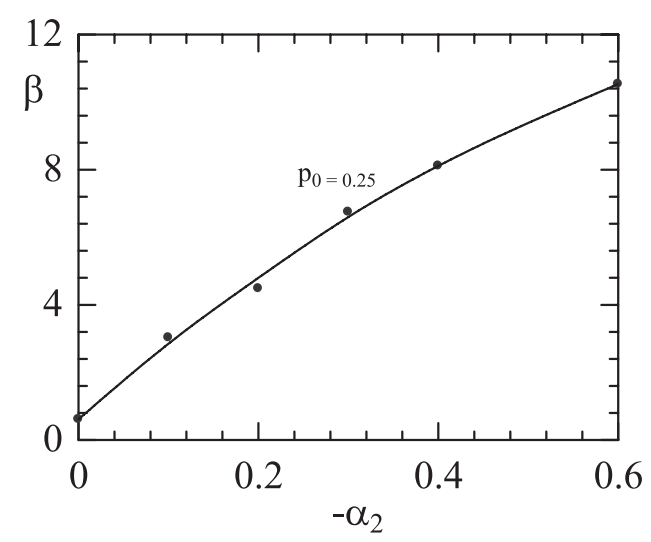

Figure 2 The expanding angle $\beta$ depending on the rheological coefficient $\alpha_{2}$ for fixed $P_{0}=0.25$.

$$
\begin{aligned}
P_{0}= & \frac{p_{g}-p_{0}}{\mu u_{*}} h_{0}=-\frac{2}{\left(1+h_{0}^{\prime 2}\right)}\left[h_{0}^{\prime 2} \frac{\partial U}{\partial \xi}-h_{0}^{\prime}\left(\frac{\partial W}{\partial \xi}+\frac{\partial U}{\partial \varsigma}\right)+\frac{\partial W}{\partial \varsigma}\right] \\
& +\frac{1}{\left(1+h_{0}^{\prime 2}\right)}\left[h_{0}^{\prime 2} \Pi_{x x}-2 h_{0}^{\prime} \Pi_{x z}+\Pi_{z z}\right]+\frac{h_{0}}{C a \cdot R_{0}} .
\end{aligned}
$$

Here $p_{g}$ and $p_{o}$ are the gas pressure outside the jet and the pressure at the point $\left(x=0, z=h_{o}\right)$, respectively, and $R_{o}$ is the free surface curvature radius at $x=0$. The capillary number is defined as

$$
C a=\frac{\sigma_{T} \Delta T}{\sigma} .
$$

The expansion of the angle depends on the rheological coefficient and is given in Figure 2.

Differences in normal stresses in the non-Newtonian fluid may increase the angle of expansion [4], and the thermocapillary effect may also increase this angle. For a fixed rheological coefficient $\alpha_{2}$, the numerical simulation provides a threshold for the expanding angle $\beta_{c}$, and the problem can be solved only if the expanding angle is larger than this threshold. Figure 3 gives the temperature distributions (left) and the counter plots of the streamlines (right), respectively, for the cases of $\alpha_{2}=0$ (upper), -0.3 (middle), -0.6 (lower), and pressure $P_{0}=0.25$. The corresponding angles of expansion are $\beta=0.61^{\circ}, 6.75^{\circ}$, and $10.53^{\circ}$, respectively. The counter plots of the streamlines show that the occurrence of a reverse flow appears to be due to the large expansion that occurs in non-Newtonian fluids. It should be noted that for better illustration, the scale in the abscissa of Figure 3 is greatly enlarged, appearing 10 times larger than reality. The ratio of the real sizes is 50 (longitu- 


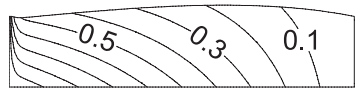

(a)

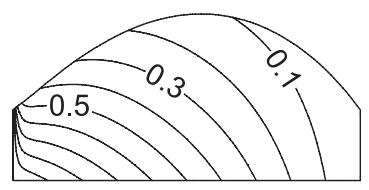

(b)

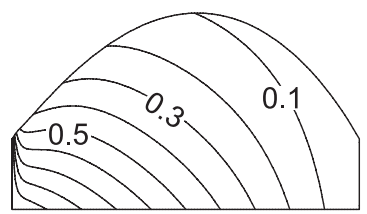

(c)

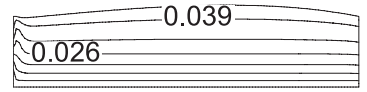

(d)

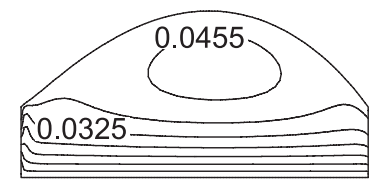

(e)

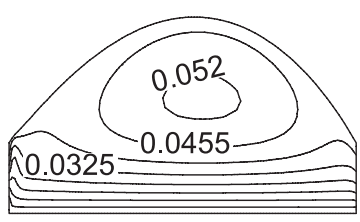

(f)

Figure 3 Temperature distributions (left) and counter plots of stream lines (right) in the cases of Newtonian fluid $\alpha_{2}=0$ (upper), non-Newtonian $\alpha_{2}=-0.3$ (middle), and -0.6 (lower) related to expanding angles $\beta=0.61^{\circ}(\mathrm{a}, \mathrm{d}), \beta=6.75^{\circ}(\mathrm{b}, \mathrm{e}), \beta=10.53^{\circ}(\mathrm{c}, \mathrm{f})$, respectively.

dinal to vertical direction), whereas the ratio of the coordinates in Figure 3 is approximately 5 .

The non-dimensional maximum heights of the cross section of the jet depend on the non-dimensional rheological coefficient $-\alpha_{2}$ and are shown in Figure 4 in the regime of $\beta \geq \beta_{c}$. Relationship $\alpha_{2}=0$ means that only the thermocapillary effect is considered. In this case, the threshold of the angle of expansion is $\beta_{c}=0.4^{\circ}$ and $\eta_{\max }=8 \%$, and is represented by the lowest real curve in Figure 4 . In our calculations, a stable solution will be reached when the angle of expansion is equal to or larger than the threshold one. One possible explanation for this result is that liquid jetting from a nozzle is in a state of constant expansion.

It should be noted that the critical curve is in direct relation to different pressures $P_{0}$ that exist for different rheological coefficients $\alpha_{2}$. Variations in maximum heights with relation to different angles of expansion, which are in turn related to the rheological coefficient $\alpha_{2}$ for a fixed pressure $P_{o}=0.25$, are shown as the dashed lines in Figure 4. However, it should be noted that even in the cases of Newtonian fluids $\left(\alpha_{2}=0\right)$, where the cross-section expansion is 


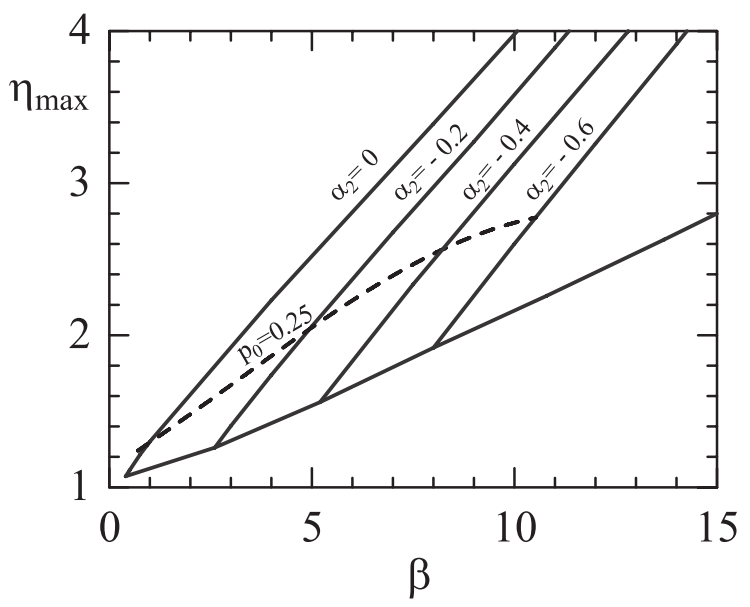

Figure 4 Maximum height of the cross section in a liquid jet depending on the expanding angle $\beta=\tan ^{-1}\left[\eta^{\prime}(0)\right]$ for different rheological coefficients $\alpha_{2}$, and the lowest real curve giving the threshold of an expanding angle depending on $\alpha_{2}$.

caused by the thermocapillary effect only, the maximum heights of the cross section may be increased 3 times if the expanding angle $\beta$ is rather large. Moreover, the rheological effect does exist in the cases of $\alpha_{2}<0$.

Thus it can be concluded that both thermocapillary and rheological effects contribute to the cross-section expansion of a jet. Additionally, the cross section can be enlarged significantly when the angle of expansion exceeds its threshold and there are strong rheological effects. In general, smaller values of $\alpha_{2}$ will result in a stronger rheological effect and a larger size for the cross section.

\section{Discussion}

The present paper deals with the simulation of a liquid jet film of nonNewtonian fluid. The simulation shows that the thermocapillary effect enlarges the cross section of the fluid jet, as a result of a heat transfer that occurs from the high temperature of a liquid jet through the free surface to the low temperature of a surrounding gas. The stress distributions depend mainly on the rheological property of the non-Newtonian fluid, which also acts to enlarge the cross section of the fluid jet. Therefore, this cross-section expansion results from the coupled action of both thermocapillary and rheological effects; however, the latter (the rheological effect) is generally more important than the former in cases where there is a threshold for an expanding angle, as discussed in [12]. 

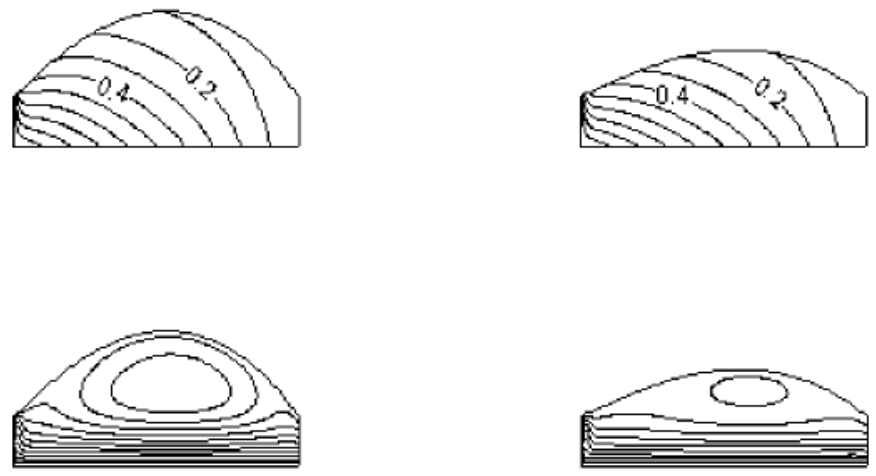

Figure 5 The temperature distributions (upper panels) and flow patterns (lower panels) for constant viscosity (left, expanding angle: 10.534 ; hmax: 2.778 ) and variable viscosity (right, expanding angle: 8.32 ; hmax: 1.984$)\left(\alpha_{2}=-0.6, P_{0}=0.25\right)$.

The results of the present paper show that, for a fixed $P_{0}$, the threshold for an expanding angle is related to various rheological effects of the fluid involved. However, the level of pressure, $P_{0}$, is sensitive to the expanding angle, and thereby related to the maximum height of the cross section, as shown in Figure 4. Similar to cases involving Newtonian fluids, the heat transfer may induce thermocapillary flows and increase the cross section of the fluid jet. Additionally, the cross section will be further enlarged if the effect of nonNewtonian fluids is involved.

The effects of viscosity variations related to temperature are considered in the comparative calculations. The results show that for a given $\alpha_{2}$ and $P_{0}$, since the viscosity decreases as the temperature increases, the expanding angle will be smaller than in the case where there is a constant viscosity. However, in these instances the patterns of the flow field and temperature distribution remain qualitatively unchanged. In one example, it is assumed that the viscosity decreases linearly with increasing temperature so that the viscosity reaches $30 \% \mu_{0}$ when $T=100^{\circ} \mathrm{C}\left(\mu_{0}\right.$ is the viscosity at $\left.T=25^{\circ} \mathrm{C}\right)$. The complete results of this example are shown in Figure 5.

The present paper adopts a second-order fluid model for non-Newtonian fluids, and thus has obvious limitations in terms of application. However, it involves a variety of polymers, and even the Newtonian fluid can describe some kinds of polymers. The conclusions of the present paper could be reasonably applied to situations involving these varieties of polymers, which can be approximately described by the second-order fluid model. For example, the Barus effect may result in a large variation in the size of a jet's cross section, and the results of the present paper may be taken as a reasonable explanation. 


\section{Acknowledgement}

This research is partly supported by the National Natural Science Foundation of China (no. 10432060).

\section{References}

[1] Tucker, C.L., Computer Modeling for Polymer Processing, pp. 370-372, Hanser, New York, 1989.

[2] Silagy, D., Demay, Y., Agussant, J.F., Stationary and stability analysis of the film casting process, J. Non-Newtonian Fluid Mech., 79 (1998), 563-570.

[3] Tanner, R.I., A theory of die-swell, J. Polym. Sci., 3 (1970), 2067-2072.

[4] Bohme, G., Die swell, in: Non-Newtonian Fluid Mechanics, Ed. G. Böhme, Ch. 4.3, North-Holland, Amsterdam, 1987.

[5] Agassant, J.F., Avenas, P., Sergant, J.Ph., Carreau, P.J., Extrudate swell, in: Polymer Processing, Eds. J.F. Agassant, P. Avenas, J.Ph. Sergant, P.J. Carreau, pp. 273-276, Hanser, New York, 1991.

[6] Ramdaul, O.O., Quere, D., Thickening factor in Marangoni coating, Langmuir, 13 (1997), 2911-2914.

[7] Quere, D., Fluid coating on a fiber, Annu. Rev. Fluid Mech., 31 (1999), $347-$ 384.

[8] Hu, W.R., Imaishi, N., Thermocapillary flow in a jet of liquid film painted on a moving boundary, Langmuir, 16 (2000), 4632-4638.

[9] Hu, W.R., Imaishi, N., Thermocapillary flow in an annular liquid layer painted on a moving fiber, Int. J. Heat Mass Transfer, 43 (2000), 4457-4466.

[10] $\mathrm{Hu}, \mathrm{W} . \mathrm{R} ., \mathrm{Hu}, \mathrm{Q}$. Influence of thermocapillary flow in a liquid film jet, Acta Astronautica, 54 (2003), 53-59.

[11] $\mathrm{Hu}$, W.R., Hui, H.W., Rheological effect on thermocapillary flow of liquid film jet printed on a moving boundary, Sci. China, Ser. A, 45 (2002), 1171-1182.

[12] Tang, Z.M., Hu, W.R., Rheological flow from a die and painting on a moving solid wall, Sci. China, Ser. G, 47 (2004), 121-128.

[13] Carpenter, B.M., Homsy, G.M., High Marangoni number convection in a square cavity: part II, Phys. Fluids A, 2 (1990), 137-149.

Paper received: 2006-01-23

Paper accepted: 2006-02-13 